\title{
Multi-stream Vlasov model for the study of relativistic Weibel-type instabilities
}

\author{
A. Inglebert ${ }^{1,2}$, A. Ghizzo ${ }^{1}$, T. Reveille ${ }^{1}$, D. Del Sarto ${ }^{1}$, \\ P. Bertrand ${ }^{1}$, F. Califano ${ }^{2}$
}

February 10, 2012

${ }^{1}$ I.J.L. UMR 7163, University of Lorraine, BP 239 F-54506 Vandoeuvre les Nancy, France.

${ }^{2}$ Department of Physics, University of Pisa, Pisa, Italy

\begin{abstract}
Abstract: We describe and apply a recently proposed model [EPS 95, 45002 (2012)] using a Hamiltonian formalism for the study of Weibel-type instabilities in the relativistic limit. Taking advantage from the invariance of the generalized canonical momentum, it allows for a drastic reduction of the computational time when compared to the full Vlasov-Maxwell system of equations. Analytically, the model is exact and we recover the standard fluid dispersion relations in the case of the Weibel and filamentation instabilities, to be considered as out benchmark physical problems.
\end{abstract}

\section{Introduction}

In these last decades, an intense research activity has been carried out for the study of the Weibel and Current Filamentation Instability (hereafter WI and CFI). Indeed, both instabilities are considered as basic processes in a plasma since associated to the possibility of generating "quasi-static" magnetic fields. The WI, first proposed by Weibel [1] (1959), is driven by the presence in the plasma of a thermal anisotropy, for example in the form of an electron biMaxwellian plasma with $T_{\perp}>T_{\|}$, where $\perp$ and $\|$ represent the perpendicular 
and parallel direction with respect to the wave vector $\mathbf{k} \equiv \mathbf{k}_{\|}$. In this case, the micro-currents produced by any small perturbation, rearrange through the growing waves into filaments, and separate more and more, thus selfconsistently generating a corresponding magnetic field [2].

A similar process occurs in the presence of an electron beam and its return current produced by the background plasma in order to maintain quasineutrality. Any infinitesimal transversal perturbation separates these currents that repeal each other, thus reinforcing the initial perturbation. As a result, a magnetic field similar to the Weibel case is generated by the CFI $[3,4]$. This instability plays a key role in the context of laser plasma interaction, in particular concerning the problem of fast ignition [5]. In laser-plasmas, fast electron beams are routinely produced during the propagation of an ultraintense, ultra-fast laser pulse, as shown by means of PIC simulations in the over-dense regime [6]. For an under-dense plasma, the CFI has been invoked in order to to explain the quasi-static magnetic fields observed in PIC simulations in the wake of the laser pulse [7] . The concept of CFI was first mentionned by Fried in Ref. [8] studying the propagation of counter-streaming beams.

The physical mechanisms underlying the development of both the Weibel and CF instabilities, is very similar and has to be found in the free energy stored in the initial phase space velocity anisotropy. In both cases, the initially unmagnetized system transforms the initial thermal, or kinetic, energy into magnetic energy associated to the generation of a dipolar magnetic field per wave-length. Due to the mathematical analogies, it is possible to describe both types of instability by the same analytical model based on the description of the perpendicular momentum space in terms of "streams".

Furthermore it is worth noticing that the relativistic formulation of the Weibel instability remains a complex problem since temperature anisotropy distributions cannot be easily introduced starting from the standard MaxwellJüttner distribution. This important point of the relativistic kinetic theory was first pointed out by Yoon in Ref. [9] who proposed a modified Maxwell-Jüttner distribution to include temperature anisotropy. However, a straightforward extension of the non relativistic approach to the relativistic regime led to a substantial discrepancy between theory and simulations, as recently observed in PIC simulations [10]. Thus the choice of an equilibrium distribution function in the relativistic regime is still today an important open problem. However, the main difficulties when studying the non-linear evolution of Weibel-like instabilities arise from the complexity to follow numerically the dynamics in phase space, in particular in the relativistic regime where the standard splitting scheme [11] cannot be used. 
Our aim here is to apply a new kinetic model, recently proposed in Ref. [12] making analytical calculations more tractable with respect to the full Vlasov-Maxwell system, but capable of representing the evolution of any anisotropic phase space distribution starting from a general initial condition. The model is here extended and apply to characterize the linear and nonlinear regimes of the instability till saturation. Such a detailed description of the different regimes is afforded by following each stream separately. Furthermore from a numerical point of view, the method also allows for a drastic reduction of the computational time.

We adopt a kinetic description and we compare our results with those obtained by solving the full Vlasov-Maxwell system of equations in the case of the CFI (see [13] and refs. therein) and of the WI [14]. The model, based on a Hamiltonian reduction technique, makes use of a multi-stream description in the limit of a one-dimensional plasma, where exact canonical invariants can be defined in the transverse direction to reduce the dimension of the momentum space (here $\mathbf{p}_{\perp}$ ). The use of such invariants allows to find out a broad class of exact non linear solutions of the Vlasov-Maxwell system. Our approach is similar to the one presented in Ref. [15] for a drift-kinetic model and in Ref. [16] for the study of magnetic reconnection. Finally, we note that since we are limited to a one-dimensional plasma, the coupling with the purely electrostatic two-stream instability is excluded.

The paper is organized as follows. In Section II we present the multistream model as derived from the Vlasov-Maxwell system. In Section III we recover the dispersion relation in the relativistic, fluid regime. The nonrelativistic limit of the dispersion relation is discussed in Section IV where we specifically address the case of the CFI and of the WI. In Section V we present a numerical comparison of our multi-stream model with the full 1D-2V Vlasov-Maxwell system in the case of the CFI. A non-symmetric case of CFI is also investigated in this section, with particular emphasis on the efficiency of the model to describe the saturation mechanism of CFI in detail. Conclusions are given in Section VI.

\section{The multi-stream Vlasov-Maxwell model}

We consider a collisionless plasmas and we adopt a kinetic description since, at least concerning the instability saturation mechanism, kinetic effects dominate and are expected also to play a key role in the further non linear dynamics. We assume, as first, the ions as a fixed neutralizing background. The Vlasov 
equation, self-consistently coupled to the Maxwell's equations, reads:

$$
\begin{gathered}
\frac{\partial F}{\partial t}+\frac{p_{x}}{m_{e} \gamma} \frac{\partial F}{\partial \mathbf{q}}+e\left(\mathbf{E}+\frac{\mathbf{p} \times \mathbf{B}}{m_{e} \gamma}\right) \frac{\partial F}{\partial \mathbf{p}}=0 \\
\gamma=\sqrt{1+\frac{p^{2}}{m_{e}^{2} c^{2}}}
\end{gathered}
$$

where $F(\mathbf{q}, \mathbf{p}, t)$ is the electron distribution function and $\gamma$ is the Lorentz factor. Here we limit our study to wave modes propagating along the $x$-direction and to a $2 \mathrm{~V}\left(p_{x}, p_{y}\right)$ momentum space with a magnetic field directed along the $z$-axis. In this case, the Vlasov equation possess an exact invariant giving the possibility to reduce the dimension of the momentum space. In our model, introduced in its main aspects in Ref. [25], the possibility of describing "exactly" the full dynamics in a reduced phase space is achieved by using a finite number $N$ of "streams" (or bunches) of particles, whose dynamics is described by a kinetic Vlasov-type equation, independent of the transverse kinetic momentum of coordinate $\mathbf{p}_{\perp}$. In the following, we discuss in detail the main points of the model . We start by considering the Hamiltonian of one particle,

$$
H=m_{e} c^{2}(\gamma-1)+e \phi(x, t)
$$

where $\phi$ is the electrostatic potential, $m_{e}$ the electron rest mass and $e$ the electron charge. By using the canonical momentum $\mathbf{P}_{c}=\mathbf{p}+e \mathbf{A}_{\perp}$, where $\mathbf{A}_{\perp}$ is the vector potential, the Lorentz factor, as defined in Eq. (2), can be expressed as

$$
\gamma=\left[1+\frac{\left(\mathbf{P}_{c}-e \mathbf{A}_{\perp}(x, t)\right)^{2}}{m_{e}^{2} c^{2}}\right]^{1 / 2}
$$

Now, along the longitudinal $x$-direction, the canonical momentum simply reduces to $P_{c, x}=p_{x}$, while in the perpendicular direction to $\mathbf{P}_{c, \perp}=\mathbf{p}_{\perp}+e \mathbf{A}_{\perp}$ (note that the choice $\mathbf{A}=\mathbf{A}_{\perp}$ corresponds to the choice of a Coulomb gauge $\nabla \cdot \mathbf{A}=0)$. Thus the Hamilton equation $\partial \mathbf{P}_{c} / \partial t=-\partial H / \partial \mathbf{q}$ can be split into the longitudinal and perpendicular direction:

$$
\begin{gathered}
\frac{d P_{c x}}{d t}=-\frac{\partial H}{\partial x} \\
\frac{d \mathbf{P}_{c, \perp}}{d t}=-\frac{\partial H}{\partial \mathbf{q}_{\perp}}=0
\end{gathered}
$$


since the Hamiltonian, as defined in Eq. (3), does not depend on the transverse spatial coordinates.

Owing to the invariance of the generalized canonical momentum, we can represent the plasma evolution by a class of initial conditions invariant under the dynamics. Without loss of generality, we represent the plasma in term of $N$ bunches of particles, each "stream" $j, j=1, \ldots, N$, having the same initial perpendicular momentum $\mathbf{P}_{c, \perp}=\mathbf{C}_{j}=$ const.

Using Eq. (5), we consider, for each $j$-th particle population, a distribution function $f_{j}\left(x, p_{x}, t\right)$ that must satisfy a reduced Vlasov-type equation. The Hamiltonian of a particle belonging to the $j$-stream, is given by:

$$
H_{j}=m_{e} c^{2}\left(\gamma_{j}-1\right)+e \phi(x, t)
$$

From Eq. (4), the $\gamma_{j}$ Lorentz factor for the $j$-stream now reads:

$$
\gamma_{j}=\left[1+\frac{p_{x}^{2}}{m_{e}^{2} c^{2}}+\frac{\left(\mathbf{C}_{j}-e \mathbf{A}_{\perp}(x, t)\right)^{2}}{m_{e}^{2} c^{2}}\right]^{1 / 2}
$$

The $j$-stream is described by the distribution function $f_{j}$ that must satisfy a "reduced" Vlasov equation:

$$
\frac{D f_{j}}{D t}=\frac{\partial f_{j}}{\partial t}+\left[f_{j}, H_{j}\right]=0
$$

where $\left[f_{j}, H_{j}\right]$ represents the standard Poisson bracket. By casting Eqs. (7)(9), we obtain the reduced Vlasov-type equation for the $j$-stream:

$$
\frac{\partial f_{j}}{\partial t}+\frac{p_{x}}{m_{e} \gamma_{j}} \frac{\partial f_{j}}{\partial x}+\left(e E_{x}-\frac{1}{2 m_{e} \gamma_{j}} \frac{\partial}{\partial x}\left(\mathbf{C}_{j}-e \mathbf{A}_{\perp}\right)^{2}\right) \frac{\partial f_{j}}{\partial p_{x}}=0
$$

These $N$ equations, representing what we defined as the multi-stream model, must be now coupled to the Maxwell's equations. By using the Poisson equation in the form

$$
\frac{\partial E_{x}}{\partial x}=\frac{e}{\varepsilon_{0}}\left(n(x, t)-n_{0}\right)
$$

where $n$ is the density defined by

$$
n(x, t)=\sum_{j=1}^{N} \int_{-\infty}^{+\infty} f_{j}\left(x, p_{x}, t\right) d p_{x}
$$

we can cast the Maxwell equations into a single one for the perpendicular potential vector $\mathbf{A}_{\perp}$ :

$$
\frac{\partial^{2} \mathbf{A}_{\perp}}{\partial t^{2}}-c^{2} \frac{\partial^{2} \mathbf{A}_{\perp}}{\partial x^{2}}=\frac{1}{\varepsilon_{0}} \sum_{j=1}^{N} \mathbf{J}_{\perp, j}(x, t)
$$


where $\mathbf{J}_{\perp, j}$ is the perpendicular current associated with the particle population $j$, i.e. the $j$-stream, and defined by:

$$
\mathbf{J}_{\perp, j}=\frac{e}{m_{e}}\left(\mathbf{C}_{j}-e \mathbf{A}_{\perp}\right) \int_{-\infty}^{+\infty} \frac{f_{j}}{\gamma_{j}} d p_{x}
$$

In summary, our multi-stream model is given by $N$ reduced Vlasov equations of type (10) for each $j$-stream, self-consistently coupled to the electrostatic longitudinal field $E_{x}$, Eq. (11), and to the potential vector $\mathbf{A}_{\perp}$, Eq. (13). One remark must be pointed out:

The full distribution function $F\left(x, p_{x}, \mathbf{p}_{\perp}, t\right)$ can now be written as a sum of Dirac distributions:

$$
F\left(x, p_{x}, \mathbf{p}_{\perp}, t\right)=\sum_{j=1}^{N} f_{j}\left(x, p_{x}, t\right) \delta\left(\mathbf{p}_{\perp}-\left(\mathbf{C}_{j}-e \mathbf{A}_{\perp}(x, t)\right)\right)
$$

thus, reducing the $3 \mathrm{D}$ phase space into a $2 \mathrm{D}$ one (plus $N$ values for the corresponding $\mathbf{C}_{j}$ ).

In the case of a two-dimensional $(x, y)$ plasma, the $z$-direction is thus reduced to the $p_{z}$ component perpendicular to the $(x, y)$ plane. Since the corresponding Hamiltonian does not depend on the variable $z$, as $F$ indeed, it is possible to find a canonical invariant $P_{c, z}=p_{z}+e A_{z}$ with a potential vector of the type $\mathbf{A}=A_{z}(x, y, t) \mathbf{e}_{z}$. Thus, the condition we need to make use of the conservation of the transverse canonical momentum to reduce the phase space dimension, is the possibility to separate the electrostatic and electromagnetic contributions in the electric field $\mathbf{E}=-\partial \mathbf{A} / \partial t-\nabla \phi$.

\section{The multi-stream model in the fluid approxi- mation}

We now derive the dispersion relation of Weibel-type instabilities in the fluid limit making use of the multi-stream model. We consider in Eq. (15), the case $N=1$, corresponding to a cold plasma distribution function in the perpendicular direction. This limit has been intensively investigated in the context of laser-plasma interaction, in particular for the study of parametric Raman-type instabilities in the semi-relativistic [17] and strongly relativistic regime [18], or in the study of self-induced transparency in over-dense plasmas [19].

The "multi-fluid" model is obtained by taking the moments of the Vlasov equation, Eq. (10). Let's now introduce, for each $j$-stream, the density $n_{j}$, the 
averaged longitudinal momentum $u_{j}$ and a mean Lorentz factor $\widetilde{\gamma_{j}}$. Without loss of generality, we assume a linearly polarized electromagnetic wave. We can write the potential vector and the transverse canonical momentum in the form:

$$
\mathbf{A}_{\perp}=A_{y}(x, t) \mathbf{e}_{y} ; \quad \mathbf{P}_{c, \perp}=P_{c, y} \mathbf{e}_{y}=\text { const }=C_{j} \mathbf{e}_{y}
$$

which corresponds to the most general case due to the $x$-axis symmetry. Here $C_{j}$ is a constant. For each $j$-stream, the continuity, motion and Poisson equations can be written as:

$$
\begin{gathered}
\frac{\partial n_{j}}{\partial t}+\frac{\partial}{\partial x}\left(\frac{n_{j} u_{j}}{m_{e} \widetilde{\gamma}_{j}}\right)=0 \\
\frac{\partial u_{j}}{\partial t}+\frac{u_{j}}{m_{e} \widetilde{\gamma_{j}}} \frac{\partial u_{j}}{\partial x}=e E_{x}-\frac{1}{2 m_{e} \widetilde{\gamma}_{j}} \frac{\partial}{\partial x}\left(C_{j}-e A_{y}\right)^{2} \\
\frac{\partial E_{x}}{\partial x}=\frac{e}{\varepsilon_{0}}\left(\sum_{j=1}^{N} n_{j}(x, t)-n_{0}\right)
\end{gathered}
$$

Using Eq. (13), we obtain the following equation for the vector potential:

$$
\left(\frac{\partial^{2}}{\partial t^{2}}-c^{2} \frac{\partial^{2}}{\partial x^{2}}+\sum_{j=1}^{N} \frac{e^{2}}{m_{e} \varepsilon_{0}} \rho_{j}\right) A_{y}=\frac{e}{m_{e} \varepsilon_{0}} \sum_{j=1}^{N} C_{j} \rho_{j}
$$

where $\rho_{j}$ is the "stream" density defined as:

$$
\rho_{j}=\int_{-\infty}^{+\infty} \frac{f_{j}}{\gamma_{j}\left(x, p_{x}, t\right)} d p_{x}
$$

Finally, the fluid limit of the Lorentz factor is obtained from Eq. (8):

$$
\widetilde{\gamma_{j}}=\left[1+\frac{u_{j}^{2}}{m_{e}^{2} c^{2}}+\frac{\left(C_{j}-e A_{y}(x, t)\right)^{2}}{m_{e}^{2} c^{2}}\right]^{1 / 2}
$$

From Eqs. (17) to (20), together with the definition of the "stream" charge density and of the "averaged" Lorentz factor, Eqs. (21)-(22), represent a closed system.

We now make a linear, normal modes analysis around an equilibrium characterized by a mean density $n_{0, j}$ (for the stream $j$ ) and a zero mean velocity $u_{j 0}$. We write the fluctuating quantities, i.e. the density, velocity, vector potential, longitudinal electric field, as $\delta n_{j}, \delta u_{j}, \delta A_{y}, \delta E_{x}$. By linearizing Eqs. 
(17) to (20) and performing a Fourier transform in time $(\partial / \partial t=-i \omega)$ and space $(\partial / \partial x=i k)$, we get the following set of equations:

$$
\begin{gathered}
-i \omega \delta n_{j}+\frac{i k n_{0, j}}{m_{e} \Gamma_{0, j}} \delta u_{j}=0 \\
-i \omega \delta u_{j}=e \delta E_{x}+\frac{i e k}{m_{e} \Gamma_{0, j}} C_{j} \delta A_{y} \\
i k \delta E_{x}=\frac{e}{\varepsilon_{0}} \sum_{j=1}^{N} \delta n_{j} \\
\left(-\omega+k^{2} c^{2}+\sum_{j=1}^{N} \frac{\omega_{p, j}^{2}}{\Gamma_{0, j}}\right) \delta A_{y}-\sum_{j=1}^{N} \frac{\omega_{p, j}^{2} C_{j}^{2}}{m_{e}^{2} c^{2} \Gamma_{0, j}^{3}} \delta A_{y}=\frac{e}{m_{e} \varepsilon_{0}} \sum_{j=1}^{N} \frac{C_{j} \delta n_{j}}{\Gamma_{0, j}}
\end{gathered}
$$

where $\omega_{p, j}^{2}=n_{0, j} e^{2} / m_{e} \varepsilon_{0}$ is the "plasma frequency" of the $j$-stream and the quantity $\Gamma_{0, j}$ corresponds to the zero-order Lorentz factor:

$$
\Gamma_{0, j}=\sqrt{1+\frac{C_{j}^{2}}{m_{e}^{2} c^{2}}}
$$

Note that, in Eqs. (25) and (26), we have imposed, at zero order, the charge neutrality condition and an initial zero net current:

$$
\begin{gathered}
\sum_{j=1}^{N} n_{0, j}=n_{0} \\
\sum_{j=1}^{N} \frac{C_{j} n_{0, j}}{\Gamma_{0, j}}=0
\end{gathered}
$$

By solving the algebraic system of equations, Eqs. (23)-(26), we finally get the dispersion relation of our multi-streams fluid system:

$$
\begin{gathered}
\left(1-\sum_{j=1}^{N} \frac{\omega_{p, j}^{2}}{\omega^{2} \Gamma_{0, j}}\right)\left\{-\omega^{2}+k^{2} c^{2}+\sum_{j=1}^{N} \frac{\omega_{p, j}^{2}}{\Gamma_{0, j}^{3}}+\frac{k^{2} c^{2}}{\omega^{2}} \sum_{j=1}^{N} \frac{\omega_{p, j}^{2}}{\Gamma_{0, j}^{3}} \frac{C_{j}^{2}}{m_{e}^{2} c^{2}}\right\} \\
=-\frac{k^{2} c^{2}}{\omega^{4}}\left(\sum_{j=1}^{N} \frac{\omega_{p, j}^{2}}{\Gamma_{0, j}^{2}} \frac{C_{j}}{m_{e} c}\right)^{2}
\end{gathered}
$$

The analytical formulation of the linear dispersion relation, Eq. (30), as a discrete summation over a collection of streams, provides a general and exact approach to take into account any anisotropy of the distribution function. 


\section{The multi-stream model in the weak relativis- tic regime}

From a mathematical point of view, the multi-stream model can be seen as a "reduction technique" of the phase space dimension, due to the elimination of the perpendicular momentum $\mathbf{p}_{\perp}$ that remains hidden in the streams $\mathbf{C}_{\perp, \mathbf{j}}$. Of course, an accurate description of a continuous distribution in $\mathbf{p}_{\perp}$, can be obtained only using a large value of $N$. However, in the case of the Weibel instability, the macroscopic description requires only the first moments (see for instance Ref. [20]), indicating that only a small number of streams is necessary to give a complete description of this instability. For the CFI, only two streams can be used to describe the instability.

The use of exact invariants simplify the analysis of a Hamiltonian system such as the Vlasov system. For example, in the $(x, \mathbf{p})$ phase space, the exchange of perpendicular momentum is described by the partial derivatives $\partial / \partial \mathbf{p}_{\perp}$. From a numerical point of view, the derivative operator $\partial / \partial \mathbf{p}_{\perp}$ has to be approximated by some finite difference scheme. Therefore a minimum size for the mesh in the perpendicular space $\mathbf{p}_{\perp}$ is required and we are faced with the usual sampling problem; on the other hand, a multi-stream description can be used even with a small number of streams, sometimes $N=2$ or $N=3$, or even with $N=1$, with a significative reduction of the numerical effort. The particular case $N=2$ corresponds, as already noted, to the current filamentation instability. In the limit of two initial "streams", $N=2$, Eq. (30) reduces to the counter-streaming instability in the relativistic regime, in agreement with Pegoraro et al. [21].

The interesting result is the fact that Eq. (30) contains only quadratic terms in $C_{j}$. Furthermore, it is always possible to define the quantity

$$
\sum_{j=1}^{N} \omega_{p, j}^{2} C_{j}^{2} / \Gamma_{0, j}^{3} m_{e}^{2} c^{2}
$$

that can be view as a "relativistic temperature". We also note that in the non relativistic regime, it is easy to construct the different streams associated to a distribution function characterized by a density $n_{0}$ and a perpendicular thermal velocity $v_{t h}$, as we will see, in the form $\sum_{j=1}^{N} \omega_{p, j}^{2} C_{j}^{2} / m_{e}^{2}=\omega_{p}^{2} v_{t h}^{2}$, allowing one, for example, to recover exactly the Weibel dispersion relation. Finally, from Eq. (30) we point out that:

(i) It is possible to recover the non relativistic regime by taking the limit $\Gamma_{0, j} \rightarrow 1$ in Eq. (30). Thus, in a multi-stream framework, the non relativistic 
dispersion relation of a Weibel-type instability is given by:

$$
k^{2} c^{2}+\omega_{p}^{2}-\omega^{2}+\frac{k^{2} c^{2}}{\omega^{2}} \sum_{j=1}^{N} \frac{\omega_{p, j}^{2} C_{j}^{2}}{m_{e}^{2} c^{2}}=0
$$

This equation shows that the longitudinal, purely electrostatic, and the transverse, purely electromagnetic, contributions are decoupled.

(ii) We can discretize the streams "coarsely", since there is no exchange of particles between the different populations, as known for other Hamiltonian systems, as for example in the water bag model [22, 23] or in the contour dynamics technique [24].

In the following, we consider two basic examples of instability in the non relativistic regime and in the approximation of a cold longitudinal plasma temperature.

\subsection{The current filamentation instability}

We take the limit $N=2$. Following Eq. (15), it is possible to represent the initial distribution function in the following form:

$$
F\left(x, p_{x}, p_{y}, t=0\right)=n_{0} \delta\left(p_{x}\right) G\left(p_{y}\right)=n_{0} \delta\left(p_{x}\right) \sum_{j=1}^{2} F_{j} \delta\left(p_{y}-C_{j}\right)
$$

where the quantities $F_{j}$ are just normalization factors. We consider the case of the CFI. This instability is driven by electron momentum anisotropy in velocity space, corresponding to two counter-streaming electron beams of momentum $p_{0,1}<0$ and $p_{0,2}>0$ and densities $n_{0,1}$ and $n_{0,2}$. Quasi-neutrality imposes that the total net current must be zero, corresponding to the condition $n_{0,1} p_{0,1}+$ $n_{0,2} p_{0,2}=0$, or

$$
\sum_{j=1}^{2} n_{0, j} C_{j}=0
$$

In Fig. 1 we show a schematic representation of the function $G\left(p_{y}\right)$ as represented by the two invariants $C_{1}=p_{0,1}$ and $C_{2}=p_{0,2}$. Since the plasma density is normalized to one, $\int F\left(x, p_{x}, p_{y}, t\right) d p_{x} d p_{y}=1$, we get:

$$
\int G\left(p_{y}\right) d p_{y}=\sum_{j=1}^{2} F_{j}=F_{1}+F_{2}=1
$$


By considering the non relativistic limit, $\Gamma_{0, j}=1$, charge neutrality and zero net current conditions, Eqs. (28) and (33), reduce to

$$
n_{0,1}+n_{0,2}=n_{0} ; \quad n_{0,1} C_{1}+n_{0,2} C_{2}=n_{0,1} p_{0,1}+n_{0,2} p_{0,2}=0
$$

from which we get

$$
\sum_{j=1}^{2} C_{j}^{2} n_{0, j}=-n_{0} C_{1} C_{2}=-n_{0} p_{0,1} p_{0,2}
$$

By replacing this expression in the dispersion relation, Eq. (31), we recover the standard dispersion relation for the non relativistic CFI:

$$
\omega^{4}-\left(k^{2} c^{2}+\omega_{p}^{2}\right) \omega^{2}+\omega_{p}^{2} k^{2} c^{2} \frac{p_{0,1} p_{0,2}}{m_{e}^{2} c^{2}}=0
$$

in agreement with Refs. $[27,28]$ where the CFI dispersion relation is obtained from the fluid equations in the relativistic regime.

\subsection{The case of the Weibel instability}

By taking $N=3$ streams, it is possible to recover the Weibel dispersion relation driven by a temperature anisotropy in velocity space. In Fig. 2 we show a schematic representation of this system and we define an initial distribution function $F$ in the form of a superposition of three of streams:

$$
F\left(x, p_{x}, p_{y}, t=0\right)=n_{0} \delta\left(p_{x}\right) G\left(p_{y}\right)=n_{0} \delta\left(p_{x}\right) \sum_{j=1}^{N=3} F_{j} \delta\left(p_{y}-C_{j}\right)
$$

Let's now consider a homogeneous Maxwellian distribution function $F(\mathbf{p})$, of corresponding density $n_{0}=1$ and thermal velocity $v_{t h}=\sqrt{k_{B} T / m_{e}}$. The zero order momentum $\iint F(\mathbf{p}) d p_{x} d p_{y}=n_{0}$, in a multi-streams approach, is given by:

$$
\int G\left(p_{y}\right) d p_{y}=\sum_{j=1}^{3} F_{j}=1
$$

The first-order $p_{y}$-momentum $\iint p_{y} F(\mathbf{p}) d p_{x} d p_{y}=0$ and imposing the zero net current condition, Eq. (33), reads:

$$
\sum_{j=1}^{3} C_{j} F_{j}=0
$$


Assuming a symmetry property, i.e. $C_{2}=0$ and $F_{1}=F_{3}$, we get $C_{1}=-C_{3}$. Then, by using higher order momenta,

$$
\begin{aligned}
& \iint p_{y}^{2} F(\mathbf{p}) d p_{x} d p_{y}=n_{0} m_{e}^{2} v_{t h}^{2} \\
& \iint p_{y}^{4} F(\mathbf{p}) d p_{x} d p_{y}=3 n_{0} m_{e}^{4} v_{t h}^{4}
\end{aligned}
$$

and using Eqs. (38), (41) and (42), we obtain the following relations:

$$
\begin{aligned}
& \sum_{j=1}^{3} C_{j}^{2} F_{j}=2 F_{1} C_{1}^{2}=m_{e}^{2} v_{t h}^{2} \\
& \sum_{j=1}^{3} F_{j} C_{j}^{4}=2 F_{1} C_{1}^{4}=3 m_{e}^{4} v_{t h}^{4}
\end{aligned}
$$

By solving Eqs. (39), (43) and (44) we get $F_{1}=F_{3}=1 / 6, F_{2}=2 / 3$ and $C_{3}=-C_{1}=\sqrt{3} m_{e} v_{t h}$. By using these parameters in Eq. (31), we finally recover the well-known dispersion relation, first obtained by Weibel [1]:

$$
\omega^{4}-\left(\omega_{p}^{2}+k^{2} c^{2}\right) \omega^{2}-\omega_{p}^{2} k^{2} v_{t h}^{2} \omega^{2}=0
$$

\section{$5 \quad$ Numerical comparison between the full kinetic approach and the reduced Vlasov models}

\subsection{The Vlasov simulation (V-model)}

We now focus on the numerical study of the current filamentation instability aiming at comparing, in the 1D-2V limit, the full Vlasov - Maxwell system, Eqs. (46), (47), (48), with the reduced Vlasov-Maxwell model presented in Section 2, Eqs. (10), (11), (13). In the following, these models will be referred as the $\mathrm{V}$-model and the R-model model, respectively.

The $\mathrm{V}$-model is solved by making use of a phase space code based on a semi-lagrangian algorithm [29] that integrates the following set of equations. From Eq. (1), the 1D-2V Vlasov equation reads,

$$
\frac{\partial F}{\partial t}+\frac{p_{x}}{m_{e} \gamma} \frac{\partial F}{\partial x}+e\left(E_{x}+\frac{p_{y} B_{z}}{m_{e} \gamma}\right) \frac{\partial F}{\partial p_{x}}+e\left(E_{y}-\frac{p_{x} B_{z}}{m_{e} \gamma}\right) \frac{\partial F}{\partial p_{y}}=0
$$


self-consistently coupled to the Maxwell's equations:

$$
\begin{gathered}
\frac{\partial B_{z}}{\partial t}=-\frac{\partial E_{y}}{\partial x} \\
\frac{\partial E_{y}}{\partial t}=-c^{2} \frac{\partial B_{z}}{\partial x}-\frac{J_{y}}{\varepsilon_{0}}
\end{gathered}
$$

where the the current, in the transverse direction, is given by

$$
J_{y}(x, t)=-e \iint \frac{p_{y}}{m_{e} \gamma} F\left(x, p_{x}, p_{y}, t\right) d p_{x} d p_{y}
$$

The longitudinal component of the electric field $E_{x}$ is obtained from the Poisson equation, Eq. (11), where the electron density $n(x, t)$ is given by

$$
n(x, t)=\iint F\left(x, p_{x}, p_{y}, t\right) d p_{x} d p_{y}
$$

The R-model is integrated by solving the $j$-Vlasov equations as written in Eq. (10), with $N=2$, self-consistently coupled with the Maxwell and Poisson equations. In this case, the fields are defined in Eqs. (12) and (14) for the electron density and the current density, respectively.

A set of numerical simulations has been performed for the case of the CFI in the relativistic regime. Both models are initialized by two counterpropagating streams. The initial distribution function is composed of a biMaxwellian distribution with beam momenta centered at $\mathbf{p}_{01} / m_{e} c=-3.0 \mathbf{e}_{y}$ and $\mathbf{p}_{02} / m_{e} c=-\left(n_{0,1} / n_{0,2}\right) \mathbf{p}_{01} / m_{e} c$. The d.f. is perturbed by purely transverse magnetic fluctuations (on the magnetic $B_{z}$-component) with wave vector $\mathbf{k}_{0}=k_{0} \mathbf{e}_{x}$, perpendicular to the electron beams. For the sake of simplicity, we have chosen a symmetric case of equal beam densities, $n_{01}=n_{02}=0.5 n_{0}$, corresponding to $\mathbf{p}_{02} / m_{e} c=3.0 \mathbf{e}_{y}$.

From now on, we use the electron mass and charge, the velocity of the light, the plasma frequency and the electron skin depth as characteristic quantities to normalize the equations. The magnetic and electric fields are normalized to $\bar{B}=\bar{E}=m_{e} c \omega_{p} / e$. The numerical space domain, in dimensionless units, is $L_{x}=2 \pi$, such that the largest wave lengths are typically the most unstable and we excite initially only the largest one admitted in the numerical domain, corresponding to $k_{0}=1$. The initial magnetic perturbation, in dimensionless units, is given by:

$$
B_{z}=a_{0} \sin \left(k_{0} x\right)
$$

where $a_{0}=10^{-4}$ is the (small) initial amplitude given in dimensionless units. In the $\mathrm{V}$-model, we take an initial electron plasma temperature $T_{e}=2 \mathrm{keV}$ in 
both the $p_{x}$ and $p_{y}$ directions. In the R-model instead, only the $p_{x}$ direction has a non zero temperature and we keep the same temperature as in the $\mathrm{V}$ model. The phase space sampling in the $\mathrm{V}$-model is $N_{x} N_{p_{x}} N_{p_{y}}=256 \times 257^{2}$, i.e. $1.69 \times 10^{7}$ grid points. The phase space sampling in the R-model is $N_{x} N_{p_{x}}$ $=512 \times 513$ grid points. The time step used in both simulations is $\Delta t=0.003$.

In Fig. 3, we plot the time evolution of the magnetic energy resulting from the numerical solution of the $\mathrm{V}$-model. During the linear phase, $10<t<25$, the initial perturbation with wave vector $k=1$ grows with a growth rate $\eta_{V-\text { model }} \simeq 0.47$, in agreement with the theoretical value, $\eta_{t h}=0.47$, obtained by solving the dispersion relation of the CFI, Eq. (30). The same curve (not shown here) has been obtained by using the multi-stream R-model initialized with two streams only, i.e. $N=2$ in Eq. (30).

The relativistic dispersion relation of the CFI in a collisionless plasma [25] and its role as a driver of non linear dynamics leading to wave-breaking processes [26], has been studied many years ago in the framework of a fluidtype description involving two electron fluid populations. These results show that wave-breaking spontaneously develop during the early non linear phase of the process of generation of quasi-static magnetic fields. However, both processes in a collisionless, plasma, are saturated by particle trapping effects as shown, in the non relativistic limit, in Ref. [28], thus requiring a fully kinetic approach.

Our kinetic V-model approach confirms the occurrence of wave breaking, as shown in Fig. 4, top panel, where we plot the time evolution of the electron density at $x=\pi / 2$ corresponding to the magnetic peak location. In the bottom panel, we show the same plot for the longitudinal electric field $E_{x}$. We see that the instability has saturated, but showing the occurrence of several plasma wave breaking events characterized by strong density peaks reaching more than three times the initial density mean value.

The first wave-breaking scenario, characterized by the strongest density peak, is observed during the saturation phase, at $t \simeq 34$.3. Such event can be considered as the final explosive phase of the cavitation process started around $t \simeq 25$.

In Fig. 5, top to bottom, we show the spatial profiles at $t=30$ of the longitudinal electric field $E_{x}$, the electron density $n_{e}$ and the magnetic field $B_{z}$ in normalized units. We see the formation of two spikes (per perturbation wavelength) associated to a wave breaking process able to produce particle acceleration depending on the initial temperature. We underline that the kinetic description adopted here allows to follow in detail the non linear relativistic dynamics associated to the cavitation process. Indeed, it provides the physically correct saturation mechanism (electromagnetic particle trapping) for the 
instability as well as for wave breaking, thus stopping the local growth of the singularity. The impact of particle trapping on the d.f. shape is shown in Fig. 6 where we draw, at $t=30$, the $p_{y}\left(p_{x}\right)$ averaged distribution function in the $\left(x, p_{x}\right)\left(\left(x, p_{y}\right)\right)$ phase space, top (bottom) panel. Similar to the electrostatic case, the d.f. filaments in the $\left(x, p_{x}\right)$ phase space evolves more and more towards vortex-like configurations. However, here the electromagnetic nature of the trapping process is visible in the $x-p_{y}$ phase space, bottom panel. We see that also in $p_{y}$ the beams, concentrated around the electrostatic peaks, rotate around the maxima of the self-generated magnetic field, in agreement with non relativistic results presented in Ref. [13].

It is worth to underline that, even if the phase space dynamics evolution is quite complex, both beams maintain their initial transverse shape indicating that each beam keeps constant its initial "temperature", as a consequence of the invariance of the perpendicular canonical momentum. It is this Hamiltonian constraint that allows us to built the multi-stream model.

In Fig. 7 we show the electric field, the density and the magnetic field as in Fig. 5, but at a later time, $t=33$. We see that the peaking process of the electric field and the density has saturated creating localized wider structures, but with a reduced amplitude, around the regions where the absolute amplitude of the magnetic field is maximum. These structures have the shape of strong density cavities centered at $x_{1}=\pi / 2$ and $x_{2}=3 \pi / 2$. The electric and magnetic field are concentrated inside such cavities where the maximum value of the density at the boundaries becomes several time (more or less three times) the initial density value. In Fig. 8 we show the $\left(x, p_{x}\right)$ and $\left(x, p_{y}\right)$ phase space at $t=33$, analogous to the one shown in Fig. 6 . We see that the previous vortex structures, typical of a regime where particle trapping is dominated by the electrostatic longitudinal component, have now been replaced by a stretched pattern driven by the magnetic force. Indeed, the magnetic to the electrostatic ratio, of order one at $t=30$, has increased by a factor of five during the cavitation process.

In Fig. 9 we show the electron density and the magnetic field in the $x-t$ plane, still obtained using the $\mathrm{V}$-model. We see, at saturation, $t \simeq 30$, the formation of two main density spikes located in $x=0, \pi$. Then, the density spikes propagate at nearly constant velocity, $v \approx 0.37$, leading to the formation of two cavities delimitated by the density spikes themselves. In the cavities we observe the concentration of the $z$-component of the magnetic field with a local plateau. We note that these processes repeat periodically and occur on a characteristic time scale of the order of the inverse of the plasma frequency. The electron non linear dynamics shown in Fig. 9, is a clear indication of the lack here of a new "meta stable" Vlasov equilibrium, as instead often observed 
in the electrostatic limit in the presence of particle trapping processes leading to the formation of vortex structures in phase space. In other words, the electron population here do not tend to relax towards any asymptotic Vlasov equilibrium, as for example the so called BGK states typical of a 1D-1V nonrelativistic electrostatic system where the ions, pushed by the "static" dipolar electric field, play a key role in the long time dynamics of the system [30]. Here instead, the system evolves on the electron time scale and it is not significantly affected by the ion's motion (as observed in the same simulation, but allowing the ions to move).

\subsection{The multi-stream simulation (R-model)}

We present now the results of a numerical simulation performed with the $\mathrm{R}$ model using the same set of physical parameters as in the previous case of the $\mathrm{V}$-model simulation, Section 5.1. All quantities are in dimensionless units as in the previous $\mathrm{V}$-model case. First of all, we note that in the reduced Vlasov equation, Eq. (10), the index $j$ is nothing but a label since no differential operator is carried out on the variable $p_{y}$. What we actually do is to bunch together particles within the same stream $j$ and let each stream evolve following the reduced Vlasov equation. The different streams are coupled through the Poisson's equation (11) and the Maxwell equations, Eqs. (47) and (48). As a result, the multi-stream model, that relies on the invariance of the perpendicular canonical momentum, gives a correct description of the plasma dynamics even using a small number of streams.

In Fig. 10 we show the "averaged" electron distribution function at $t=$ $30,33,36$ in the $\left(x, p_{x}\right)$ phase space resulting from the contribution of both

beams, $\sum_{j=1,2} f_{j}\left(x, p_{x}, t\right)$. These results must be compared with the analogous ones obtained by means of the V-model and presented in Figs. 6 and 8, top panels. We see that the evolution of the d.f. iso-lines in phase space are very accurately reproduced by the R-model. However, the use of the R-model allows us to separate the specific contributions of each beam during the development of the CFI. In particular, let's now focus our attention on the dynamics of the second beam. In Fig. 11, we show the phase space distribution function of the second stream, $f_{2}\left(x, p_{x}, t\right)$, at the same times as in Fig. 10, $t=30,33,36$. By taking the zero moment of the distribution $f_{2}\left(x, p_{x}, t\right)$, we plot in Fig. 12 the corresponding electron density, i.e. the density associated to the second electron beam.

In Fig. 11 we observe the initial electron stream acquiring a $p_{x}$ momentum component, comparable to the initial $p_{y}$ one, due to the rotation around the self-generated magnetic field. At the same time, local density (and electric) 
spikes are produced by large amplitude plasma fluctuations [31], pumped by the electrostatic electric field component driven non linearly by the CFI (see Ref. [28], Fig. 6 in the non relativistic limit). These spikes last for a few plasma times at a given spatial location (see Fig. 12) before disappearing, but sufficiently long to accelerate bunch of particles at energies larger then the initial ones, eventually leading to the formation of filaments starting from the central bulk of the distribution function and winding up more and more in phase space (see Fig. 11, central and bottom panels). Thus the analysis of a specific beam (in this case the second one) allowed by the R-model shows the mechanism of particles acceleration as a consequence of wave breaking events. The particles then evolve in phase space due to the combined effects of the ballistic motion in the presence of electromagnetic forces.

\subsection{A non-symmetric case for CFI \% added sub-section $\%$}

In this section we give a last example concerning a non symmetric case of CFI instability. Identifying the magnetic field amplification mechanism and the resulting coupling with particles in the saturation regime is the key for a better understanding of CFI or Weibel-type instabilities. The small spatiotemporel scales that are accessible to global Vlasov-Maxwell simulations and the constraints on computational time and phase space sampling imply that, such global simulations can often not be applied to examine in detail both aspects of magnetic field amplification and the resulting wave-particle dynamics. Our multi-stream model can give important insight into kinetic processes leading to the saturation process itself. By selecting appropriate initial particle "bunches", the phase space structures can be isolated and examined in detail, allowing to separate the different physical processes linked to each beam.

We consider a slightly different case where the system is initially non symmetric leading to a different structure of the wave breaking process. As first, using the V-model, we have carried out a numerical simulation with parameters $n_{0,1}=0.4$ and $p_{0,1}=4$ for the first beam and $n_{0,2}=0.6$ and $p_{0,2}=-n_{0,1} p_{0,1} /\left(n_{0,2} \gamma_{0,1}\right) \simeq-0.85$ for the second counter-propagating beam. Both streams have the same temperatures, namely $T_{e x}=T_{e y}=2 \mathrm{keV}$. The phase space sampling is $N_{x} N_{p_{x}} N_{p_{y}}=256 \times 257^{2}$ in the full Vlasov simulation (the $\mathrm{V}$-model). The perturbation amplitude for the magnetic field is given by $\delta B_{0}=10^{-5}$ and we excite the same wave vector $k_{0}=1$.

In Fig 13 we draw the time evolution of the (longitudinal) electrostatic energy in top panel together with the corresponding evolution of the electron 
density measured at the middle of the box. As expected the electron density in Fig 13 reveals a successive of strong density peaks as the result of several wave breaking processes. The same simulation has been now carried out by means of the R-model and the corresponding time evolution of the electrostatic energy is plotted on top panel in Fig. 14. We observe a very good agreement with the numerical results of Fig. 13 obtained with the V-model. The oscillatory behaviour observed in the electrostatic energy takes place during the saturation phase of CFI. The bottom panel in Fig. 14 shows the standard evolution of the magnetic energy: in the linear phase the magnetic energy increases linearly (in a logarithmic scale) with a numerical growth rate of $\gamma_{\text {num }} \simeq 0.393$ in very good agreement with the theoretical value of $\gamma_{t h}=0.398$, followed by the regime of saturation till $t \geq 35$. The nature of the wave-breaking changes going from the symmetric to the non-symmetric case. The occurrence of strong wave-breaking associated with the formation of two density spikes and the occurrence of a rotating structure in phase space in the fast stream; finally, a large cavitation in the density takes place for the slow electron population.

In Fig. 15 we focus on the shaded isocontours of the electron distribution function corresponding to the fast beam (i.e. with a transverse momentum of $p_{0,1}=4$ in normalized units). The phase space structures reveal that the growing magnetic fields modify the electron trajectories of the fast beam which now becomes magnetically trapped. In this last case we have used a finer $x-p_{x}$ phase space mesh grid with $N_{x} N_{p_{x}}=513^{2}$ grid points. In Figs. 15 electrons make a global rotation between times $t=33.8$ (the vertical arrow indicates here the "initial" position of the filament around the peak of density) and the final time $t=48.8$. At that instant, the filament, located inside the magnetically trapping structure, exhibits a similar shape in comparison to the start time $t=33.8$. Thus the corresponding magnetic bounce frequency is close, in dimensionless units, to $\omega_{B} \simeq 0.40$ (or equivalently to a bounce period of $T_{B}=15.48$, i.e. the time during three successive peaks in electron density in bottom panel in Fig. 13). The simulation is thus consistent with the magnetic trapping saturation theory as suggested by Davidson et al in [32]. In particular in magnetic trapping theory the instability reaches a steady state when the magnetic bounce frequency $\omega_{B}$ becomes comparable to the linear growth rate $\gamma_{t h}$, which is the case here since $\gamma_{t h}=0.398$. The magnetic bounce frequency is here, (see Ref. [33] ) in the relativistic regime of the instability, defined by

$$
\left(\frac{\omega_{B}}{\omega_{p}}\right)^{2}=\frac{k_{0} c}{\omega_{p}} \frac{p_{01}}{m \gamma_{01}} \frac{\omega_{c e}}{\omega_{p}}
$$

where $p_{01}$ and $\gamma_{01}$ are respectively the transverse momentum and Lorentz factor 
of the first stream. Here $\omega_{c e}=e B_{\max } / m \gamma_{01}$. Using $p_{01}=4, \gamma_{01}=4.123$ and $B_{\max }=0.70$ we obtain an estimate of $\omega_{B} \simeq 0.406$ in good agreement with the numerical value obtained by the R-model. In Fig. 15 the first five curves of the distribution function correspond to the first period of the magnetic bounce motion, while the last curve, at time $t=150$, shows the trapping structure after several bounce motions (after eight temporal periods). We observe that such a structure can persist over a long time.

\section{CONCLUSION}

A multi-stream model based on a Hamiltonian reduction technique, first developped in [12], has been applied to study CFI both in the linear and nonlinear regimes of the instability till saturation. The multi-stream model is a set of kinetic Vlasov-type equations obtained in a Hamiltonian framework, allowing to reduce the dimension of the phase space, but without loosing the accuracy of the system dynamics. The idea is to suppress the perpendicular momentum variable $\mathbf{p}_{\perp}$ taking advantage from the invariance of the generalized canonical momentum, i.e. by noticing that, for a given $j$-population, the quantity $\mathbf{P}_{c, \perp}=\mathbf{p}_{\perp}+e \mathbf{A}_{\perp}=\mathbf{C}_{j}$ is constant in time when the $\mathbf{q}_{\perp}$ variable is lacking.

By approximating the system as a finite number of "streams" or, in other words, as a summation over an ensemble of Dirac distributions, we have obtained the linear dispersion relation of Weibel-type instabilities. This formulation gives also a clear description of the temperature effects in the non relativistic regime. Furthermore, by a direct comparison with the full Vlasov model, we have shown that our R-model provides a very accurate description of the non linear, relativistic regime in the case of the current filamentation instability. We underline that here, for the sake of simplicity, we have considered the case of two symmetric beams as a "typical" case for the CFI; however, the multi-stream model is not limited to such special configurations.

To conclude, the multi-stream model offers an exact description of the plasma dynamics even with a small number of "streams", providing a relatively simpler analytical framework and bringing the link between different types of instabilities, as for example the CFI and the Weibel. From a physical point of view, interesting results can be obtained even in the limit of a small number of "streams", e.g. $N=1,2$ or 3 . On the other hand, for problems where a larger $N$ is needed for an accurate description of a continuous distribution, the numerical effort becomes more and more comparable to that required for a standard Vlasov approach (defined as the V-model). However, we believe 
that the use of the multi-stream sampling should be more accurate because of the lack of numerical diffusivity in the reduced dimension direction as well as for the less important phase space filamentation effects that typically affect Vlasov Eulerian calculations.

The philosophy used in the multi-stream approach is reminiscent of the reduction procedure met in Hamiltonian theory, as for example the canonical realization of a Lie group, which is a subgroup associated with the Lie algebra composed of the ordinary Poisson bracket (see Ref [24]). The water-bag concept in Refs. $[22,23]$ or the Hamilton-Jacobi model are other examples of Hamiltonian reduction techniques. While the water-bag concept makes full use of the Liouville invariance in phase space (we follow only the evolution of phase space contours using the property $d f(d t=0)$, the Hamilton-Jacobi model is based on the property of existence of adiabatic invariants in action-angle variables when well separated time and space scales can be found. Furthermore, in the "multi-streams" model, there are no constraints on the shape of the distribution function which can be far from a Maxwellian one.

\section{Acknowledgment}

We acknowledge the IDRIS computational center, Orsay, France, for computer time allocation on their computers.

\section{References}

[1] E. W. Weibel, Phys. Rev. Lett 2, 83 (1959).

[2] R. L. Morse, C.W. Nielson, Phys. Fluids 14, 830, (1971).

[3] G.A. Askar'yan, S.V. Bulanov, F. Pegoraro, A.M. Pukhov, Comments Plasma Phys. Contol. Fus. 17, 35 (1995)

[4] A.R. Bell, J.R. Davies, S. Guerin, H. Ruhl, Plasma Phys. Control. Fus. 39, 653 (1997)

[5] M. Tabak, J. Hammer, M.E. Glinsky, W.L. Kruer, S.C. Wilks, J. Woodworth, E.M. campbell, M.D. Perry, R.J. Mason, Phys. Plasmas 1, 166 (1994)

[6] Y. Sentoku, K. Mima, S.I. Kojima, H. Ruhl, Phys. Plasmas 7, 689 (2000)

[7] T. Lisseikina, F. Califano, V. Vshivkov, F. Pegoraro, S. Bulanov, Phys. Rev. E 60, 599 (1999) 
[8] B.D. Fried, Phys. Fluids 2, 337 (1959).

[9] P. H. Yoon, Phys. Plasmas 14, 024504 (2007).

[10] H.H. Kaang, C.M. Ryu, P. Yoon, Phys. Plasmas 16, 082193 (2009).

[11] F. Huot, A. Ghizzo, P. Bertrand, E. Sonnendrücker, O. Coulaud J. Comput. Phys. 185, 512 (2003).

[12] A. Inglebert, A. Ghizzo, T. Reveille, D. Del Sarto, P. Bertrand, F. Califano, Euro. Phys. Letters 95, 45002 (2011).

[13] F. Califano, T. Cecchi, C. Chiuderi, Phys. Plasmas 9, 451 (2002).

[14] L. Palodhi, F. Califano, F. Pegoraro Plasma Phys. Control. Fus. 51, 125006 (2009).

[15] H.J. de Blank Phys. Plasmas 8, 3927 (2001).

[16] T.V.Liseikina, F. Pegoraro, E. Yu. Echkina, Phys. Plasmas 11, 3535 (2004).

[17] M. Albrecht-Marc, A. Ghizzo, T.W. Johnston, T. Reveille, D. Del Sarto, P. Bertrand, Phys. Plasmas 14, 072704 (2007).

[18] A. Ghizzo, T.W. Johnston, T. Reveille, P. Bertrand, M. Albrecht-Marc, Phys. Rev. E 74, 1 (2006).

[19] A. Ghizzo, D. Del Sarto, T. Reveille, N. Besse, R. Klein, Phys. Plasmas 14, 062702 (2007).

[20] B. Basu, Phys. Plasmas 12, 5131 (2002).

[21] F. Pegoraro, S.V. Bulanov, F. Califano, M. Lontano, Phys Scripta T63, 262 (1996).

[22] D.C. DePackh, J. Electron Control 13, 417 (1962).

[23] P. Bertrand, J.P. Doremus, G. Baumann, M.R. Feix, Phys. Fluids 15, 1275 (1972).

[24] P.J. Morrison, Phys. Plasmas 12, 058102 (2005).

[25] F. Califano, R. Prandi, F. Pegoraro, S. Bulanov, Phys. Rev. E 58, 7837 (1998). 
[26] F. Califano, R. Prandi, F. Pegoraro, S. Bulanov, J. Plasma Physics 60, 331 (1998).

[27] F. Califano, F. Pegoraro, S.V. Bulanov, Phys. Rev. E 56, 963 (1997).

[28] F. Califano, F. Pegoraro, S.V. Bulanov, A. Mangeney, Phys. Rev. E 57, 7048 (1998).

[29] A. Ghizzo, F. Huot, P. Bertrand, J. Comput. Phys. 186, 47 (2003).

[30] F. Califano, L. Galeotti, C. Briand, Phys. Plasmas 14, 052306 (2007).

[31] Davidson, R.C., 1972, Methods in linear and nonlinear plasma theory, pag. 40, Academic Press

[32] R.C. Davidson, D.A. Hammer, I. Haber, C.E. Wagner, Phys. Fluids 15, 317 (1972).

[33] H.H. Kaang, C.M. Ryu, P.H. Yoon, Physics of Plasmas 16, 082103, (2009). 


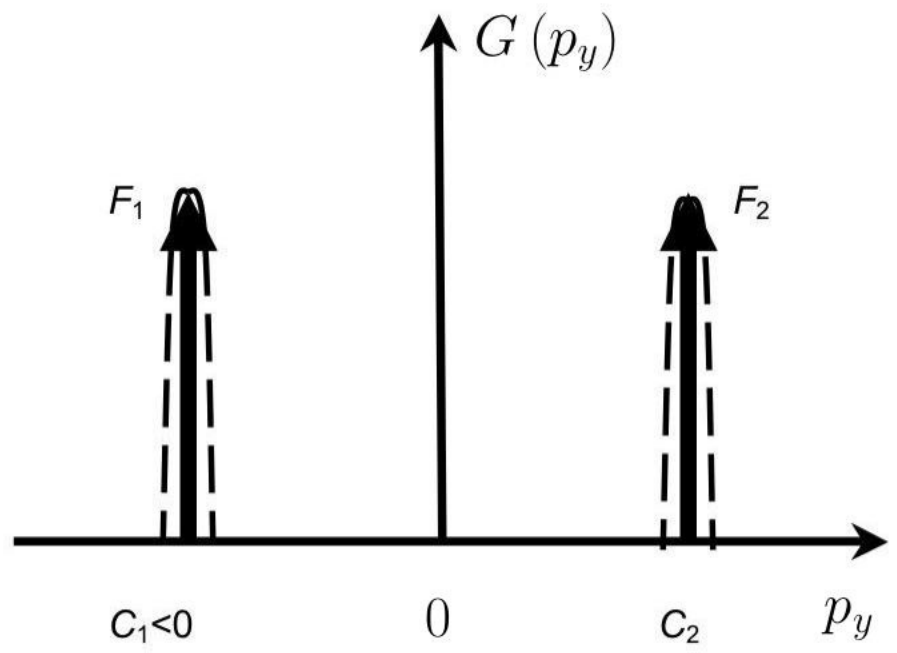

Figure 1: $G$ vs. the perpendicular momentum $p_{y}$ as represented by Dirac masses in the R-model for the case of the counter filamentation instability, in dotted lines the real shapes of the distibution function. 


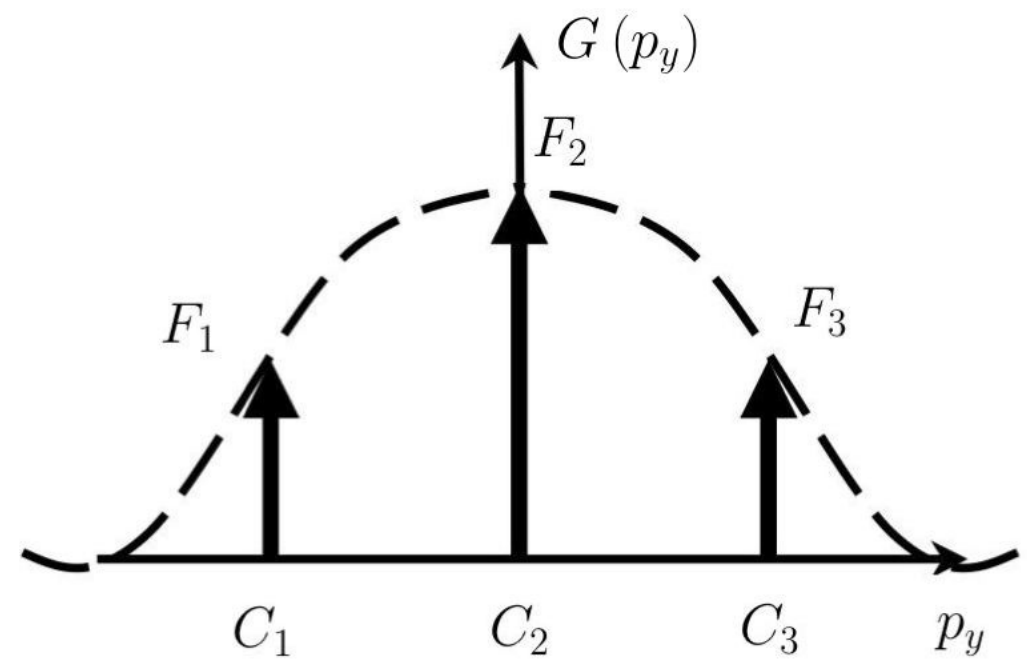

Figure 2: vs. the perpendicular momentum $p_{y}$ as represented by Dirac masses in the R-model for the case of the Weibel instability, we have represented in dotted line the shape of the distribution in the form of a Maxwellian. 


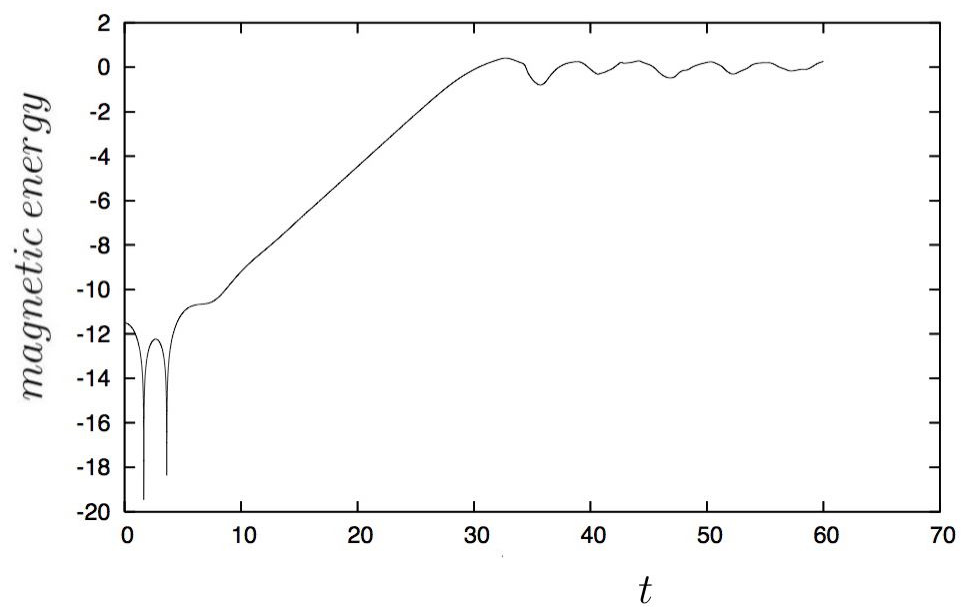

Figure 3: vs time for $k=1$ using the $\mathrm{V}$-model. The numerical growth rate, $\eta_{V-\text { model }} \simeq 0.470$, is in very good agreement with the theoretical value, $\eta_{t h}=$ 0.47 . The physical parameters are $p_{0,1 / 2}= \pm 3$ and $n_{0,1 / 2}=0.5$. All units are dimensionless. 

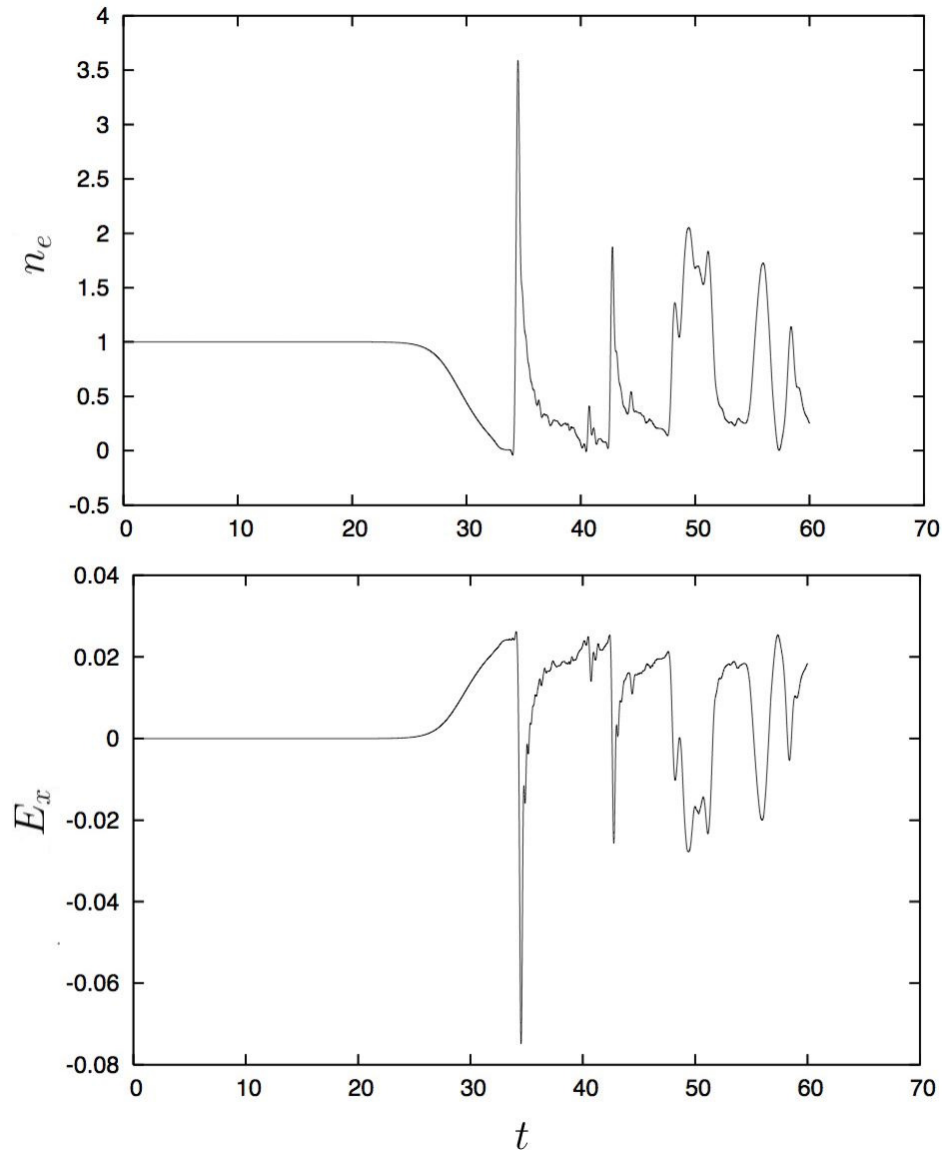

Figure 4: $n_{e}$ at $x=\pi / 2$ vs. time. Bottom panel: same plot for the longitudinal electric field $E_{x}$. 

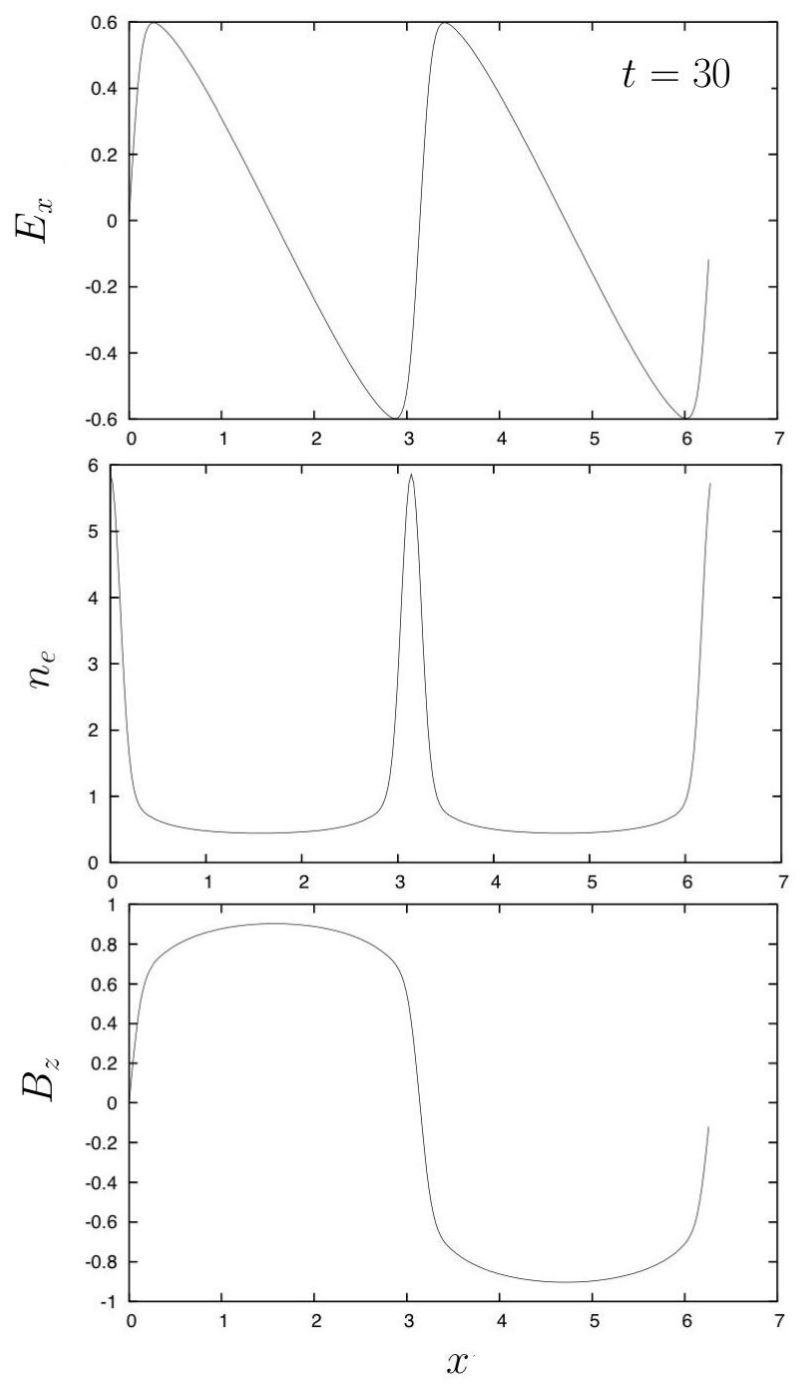

Figure 5: $E_{x}$, the electron density $n_{e}$ and the $z$-magnetic field component $B_{z}$ vs. $x$ at time $t=30$, top, middle and bottom panel, respectively, obtained with the V-model. 

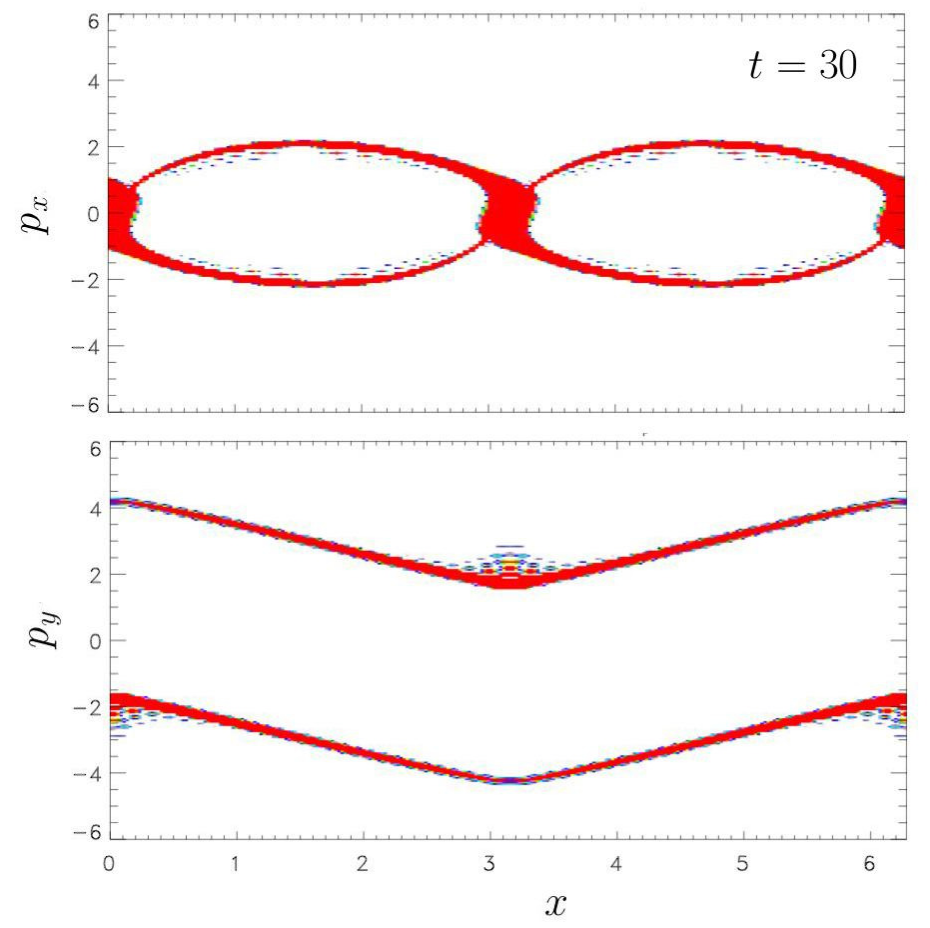

Figure 6: $\left(x, p_{x}\right)$ and $\left(x, p_{y}\right)$ phase space (top and bottom panel) averaged in the $p_{y}$ and $p_{x}$ direction, respectively, at $t=30$. These results have been obtained by using the 1D-2V Vlasov-Maxwell code (V-model) in the case of the current filamentation instability. The initial distribution condition is symmetric and is composed of two Maxwellian with beams moments centered at $\boldsymbol{p}_{0,1 / 2}= \pm 3 \mathbf{e}_{y}$. 

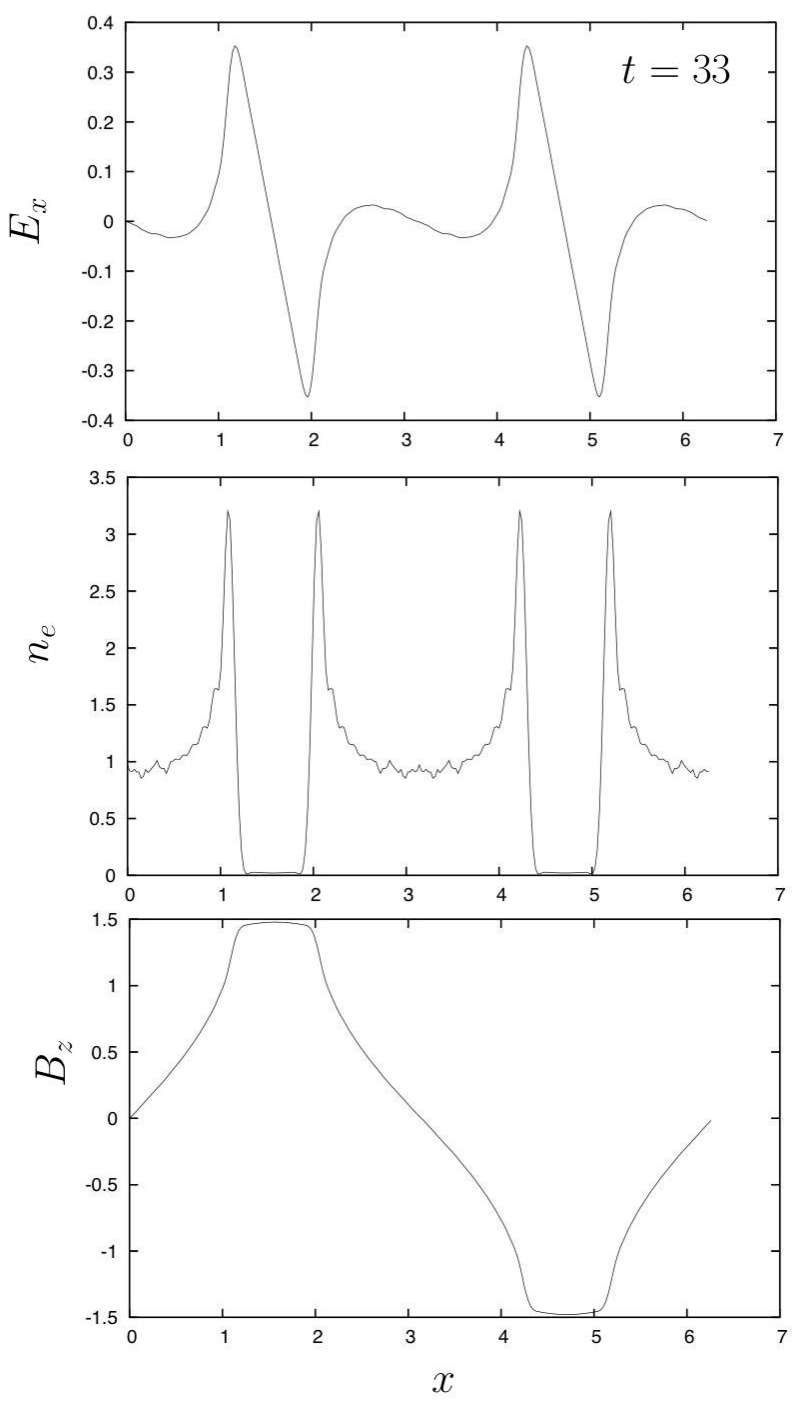

Figure 7: $E_{x}$, the electron density $n_{e}$ and the $z$-magnetic field component $B_{z}$ vs. $x$ at time $t=33$, top, middle and bottom panel, respectively, obtained with the $\mathrm{V}$-model 


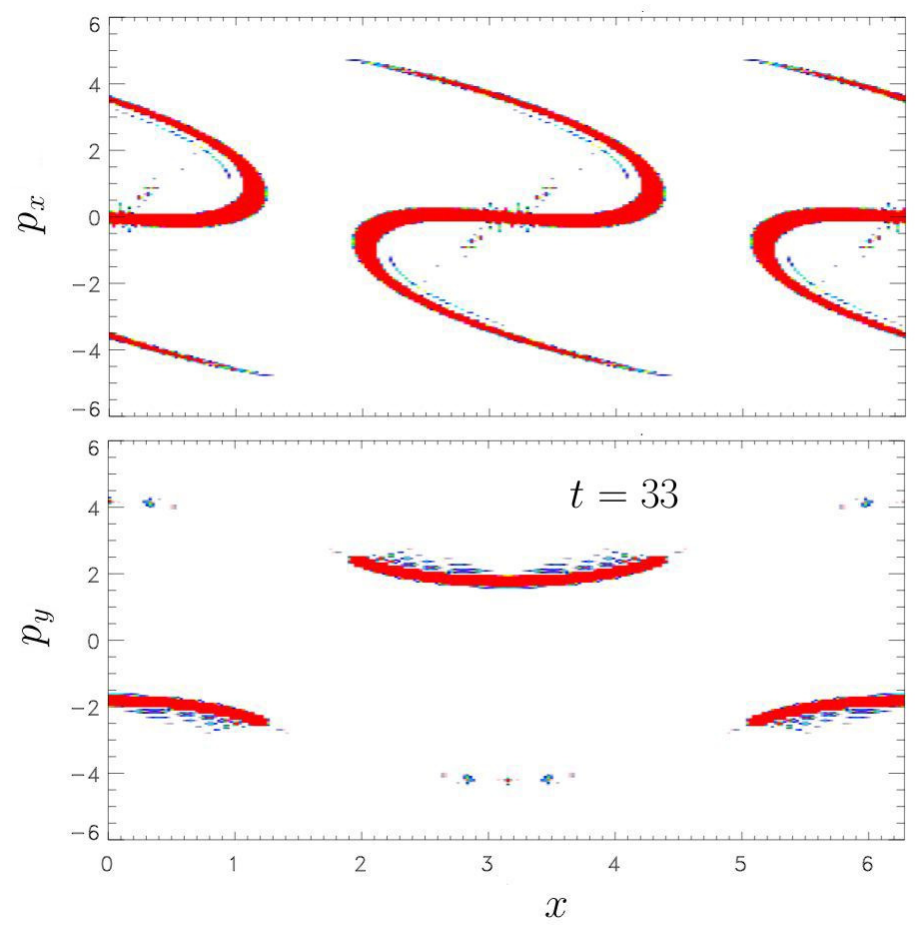

Figure 8: $\left(x, p_{x}\right)$ and $\left(x, p_{y}\right)$ phase space (top and bottom panel) averaged in the $p_{y}$ and $p_{x}$ direction, respectively, at $t=33$. These results have been obtained using the 1D-2V Vlasov-Maxwell code (V-model) in the case of the current filamentation instability 

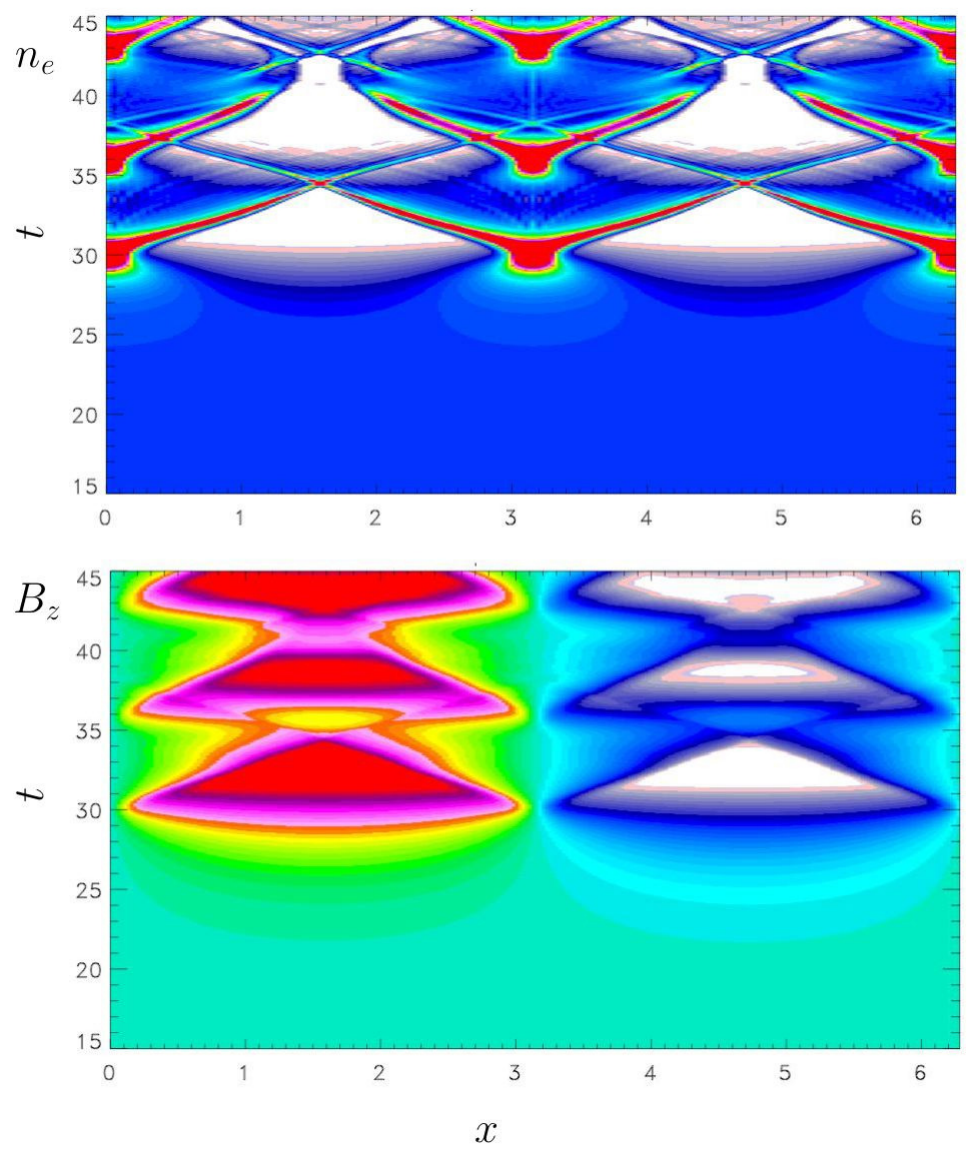

Figure 9: $n_{e}$ (top panel ) and the magnetic field $B_{z}$ (bottom ) in the $x-t$ plane obtained by means of the $\mathrm{V}$-model. 

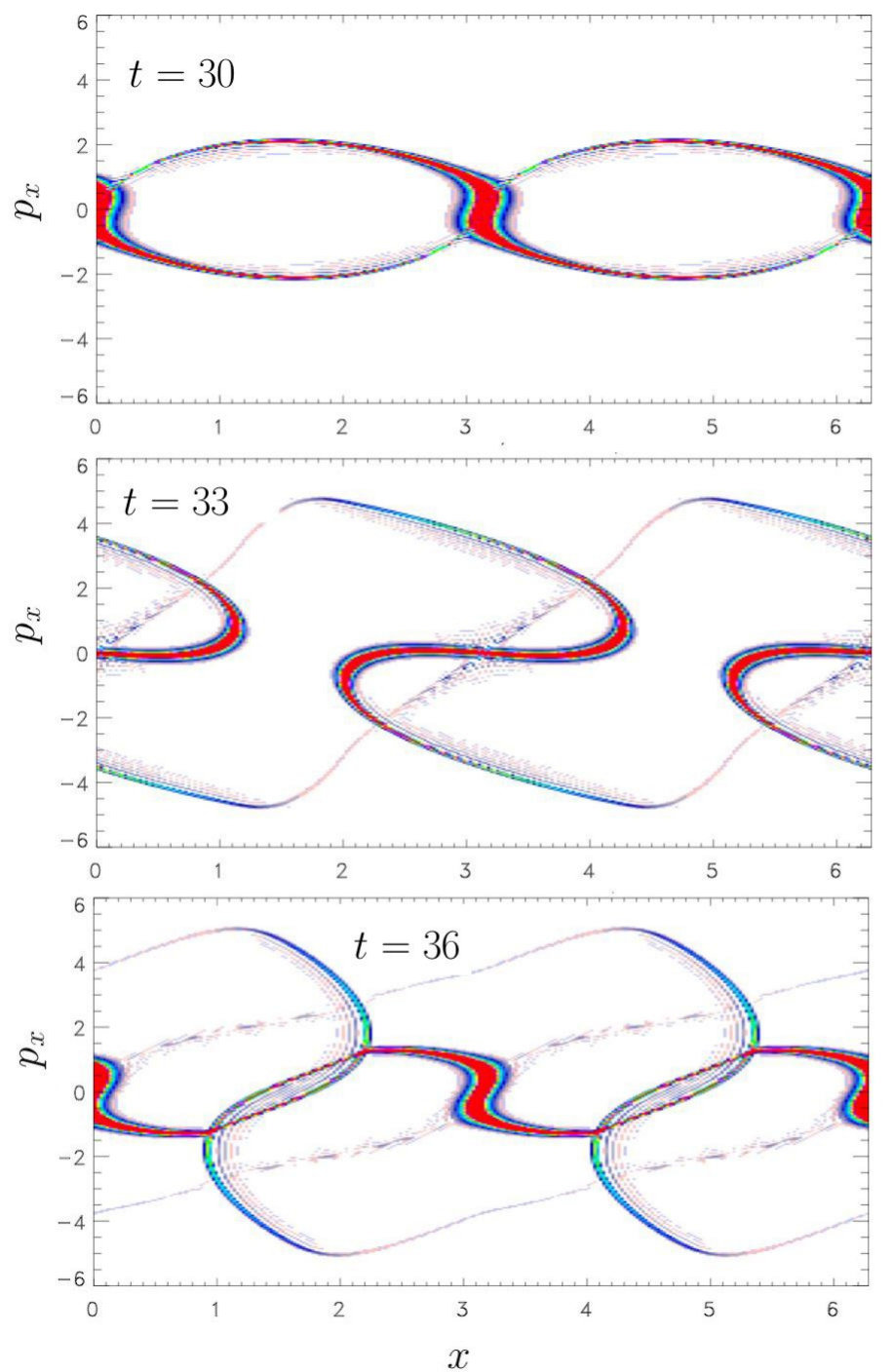

Figure 10: $\left(x, p_{x}\right)$ phase space representation of the quantity $\sum_{j} f_{j}\left(x, p_{x}, t\right)$ where the summation is made over the two streams at $t=30,33,36$. These results have been obtained by using the R-model in the case of the current filamentation instability. 

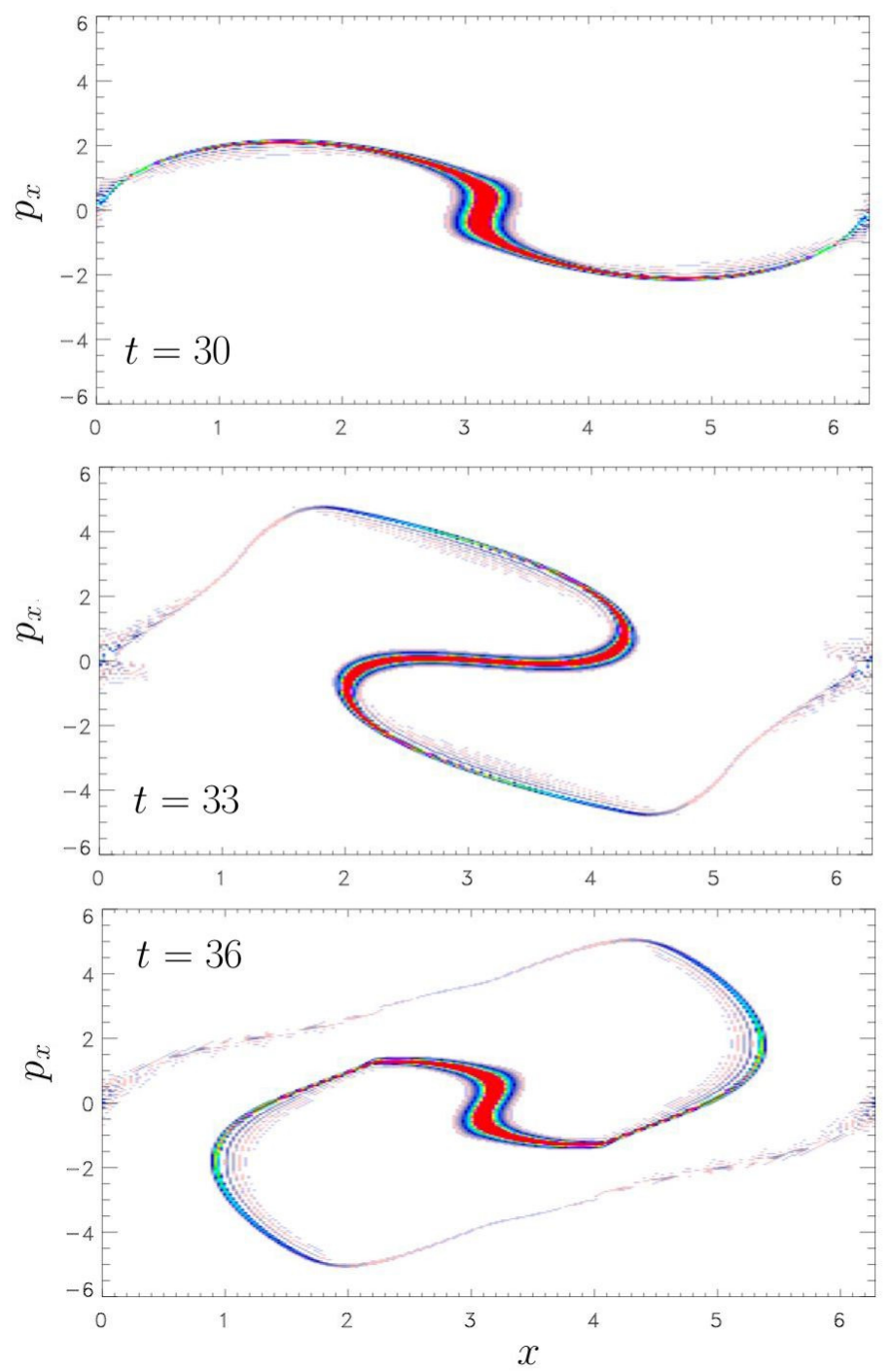

Figure 11: $\left.x, p_{x}\right)$ phase space of the second beam, $j=2$, using the R-model at $t=30,33,36$. 

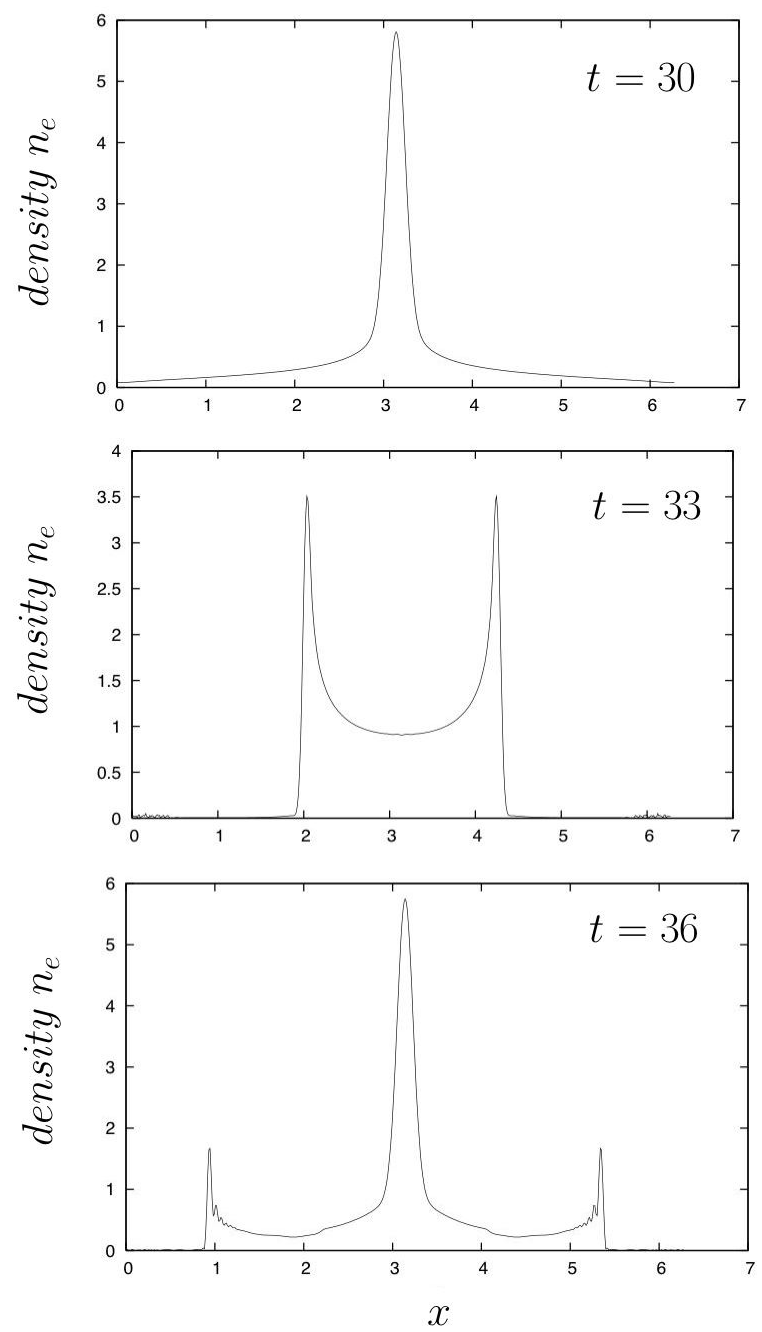

Figure 12: $j=2$, at $t=30,33,36$. 

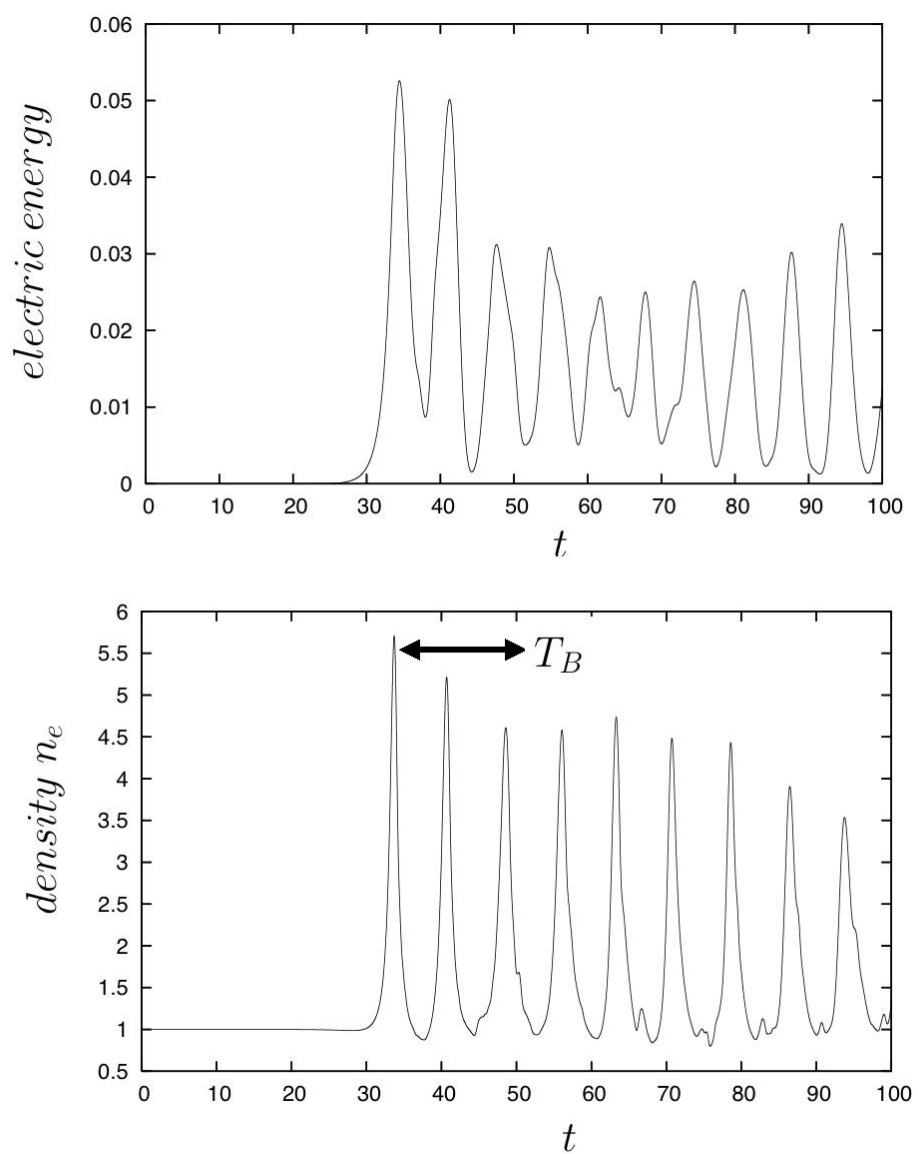

Figure 13: The electrostatic energy (top panel) and the corresponding electron density $n_{e}$ at $x=\pi v$ s. time (on bottom panel) using the $\mathrm{V}$-model. The physical parameters are $n_{01}=0.40$ and $p_{01}=4$ for the first stream and $n_{02}=0.6$ and $p_{02}=-0.86$ for the second. The arrow indicates a magnetic bounce trapping period. 

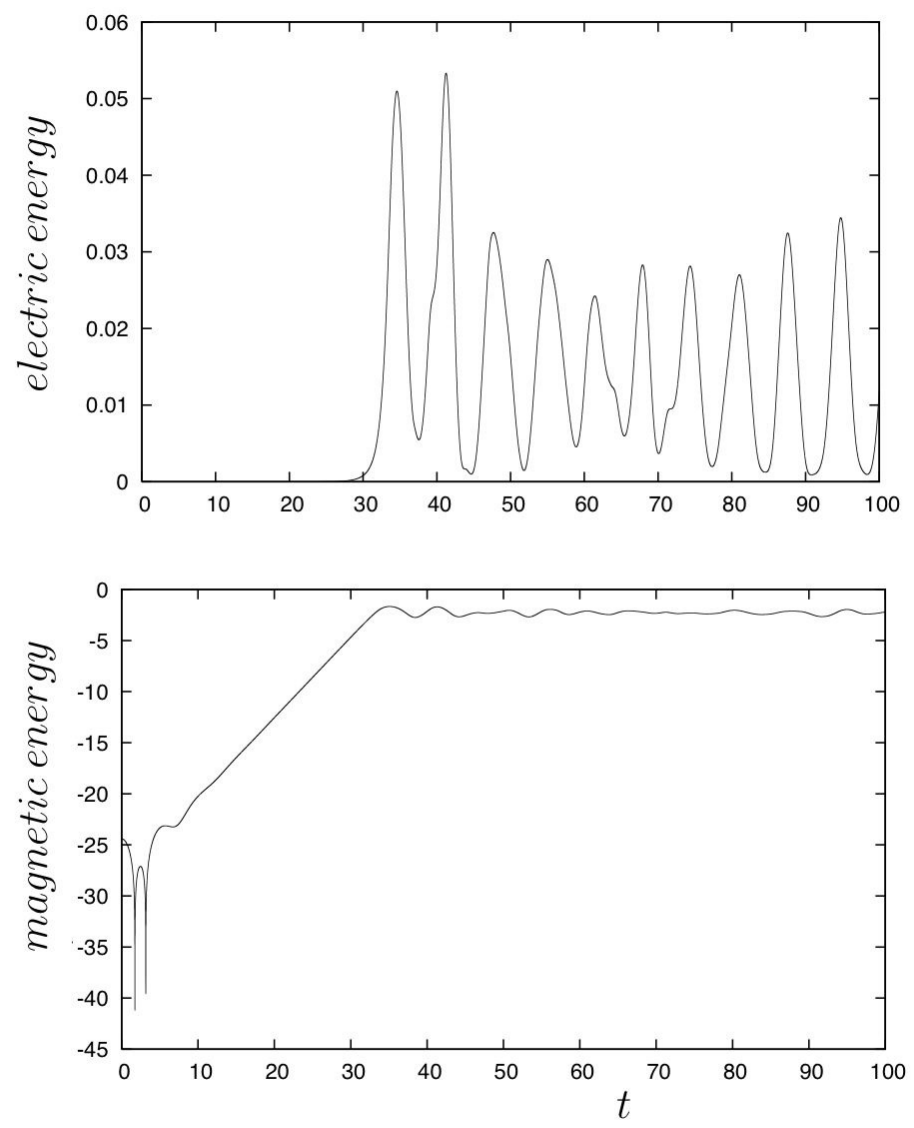

Figure 14: The electrostatic energy vs. time on top panel, using the R-model. Bottom panel: the magnetic energy in logarithmic scale vs. time. The physical parameters are identical to those used in Fig. 13. 

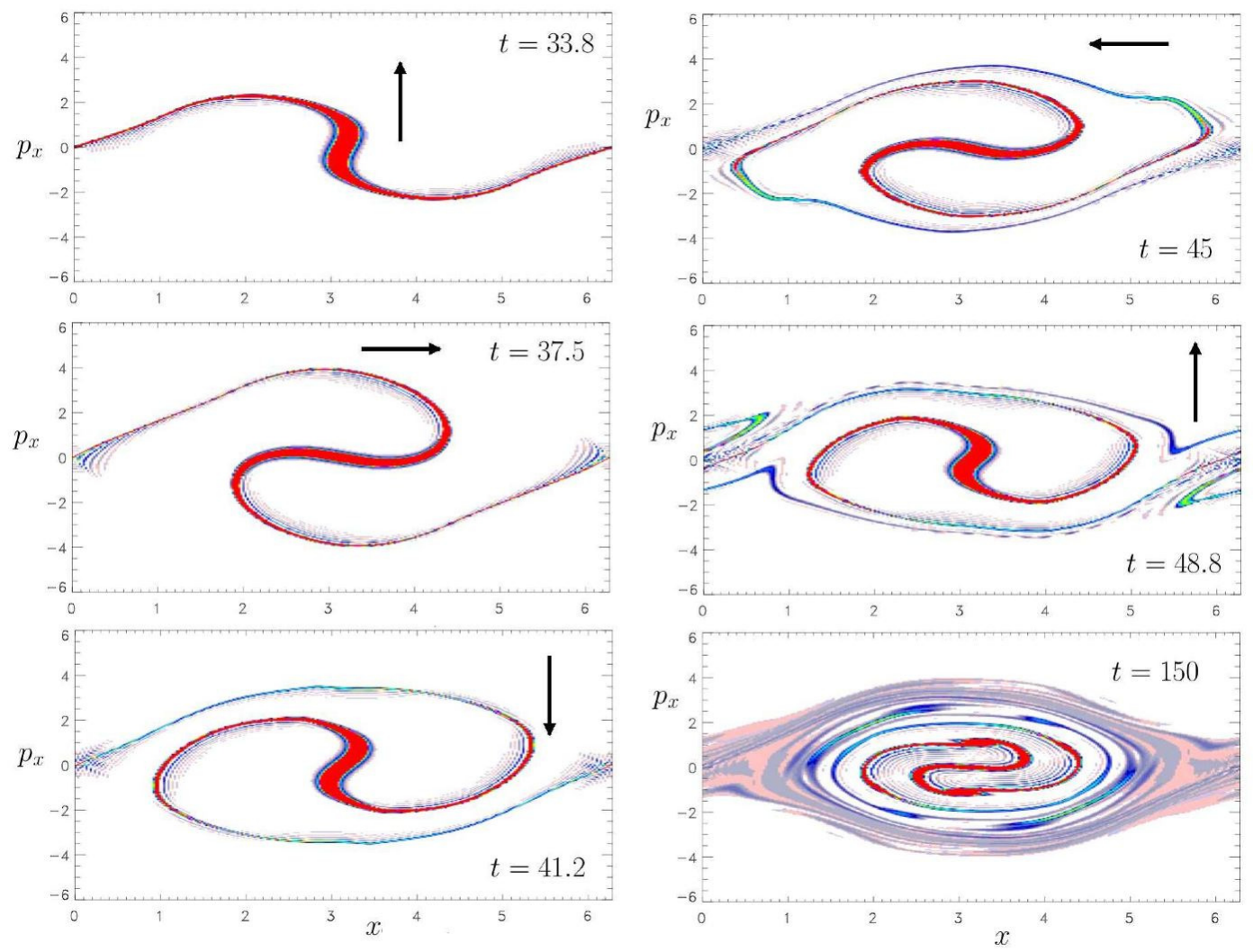

Figure 15: $x, p_{x}$ phase space of the first stream, $j=1$ (fast beam), using the R-model. The arrows indicate the position of the filament around the density peak during the rotation for the first magnetic bounce motion. Last curve, at time $\mathrm{t}=150$, shows the magnetic trapping structure after eight magnetic bounce periods. 